\title{
Ironing Out Cosmic Rays
}

\author{
A spectrometer on the International Space Station has measured the \\ energy spectrum of iron-the heaviest cosmic-ray element characterized \\ to date.
}

By Matteo Rini

$\prod$ he cosmic rays constantly bombarding Earth mostly consist of protons and helium nuclei, but they also include ions of heavier elements. By comparing the energy spectra of different cosmic-ray particles, researchers hope to garner information on the processes that forged and accelerated those particles in supernovae and on the interstellar medium through which the particles propagated. Now, the Alpha Magnetic Spectrometer (AMS) Collaboration has measured, with percent-level precision, the spectrum of iron-the heaviest element yet to be accurately characterized $[1]$.

The iron measurement is the culmination of a series of experiments aimed at characterizing the "heavy side" of cosmic rays. Obtaining accurate spectra gets increasingly tricky as the atomic number increases because heavy elements are much scarcer in the cosmos and, thus, in cosmic rays. In 2020, the AMS Collaboration measured the spectra of elements up to silicon, which has an atomic number of 14 (see Focus: New Data Reveal the Heavy Side of Cosmic Rays). Those spectra contained subtle, yet-to-be-explained differences with the ones measured

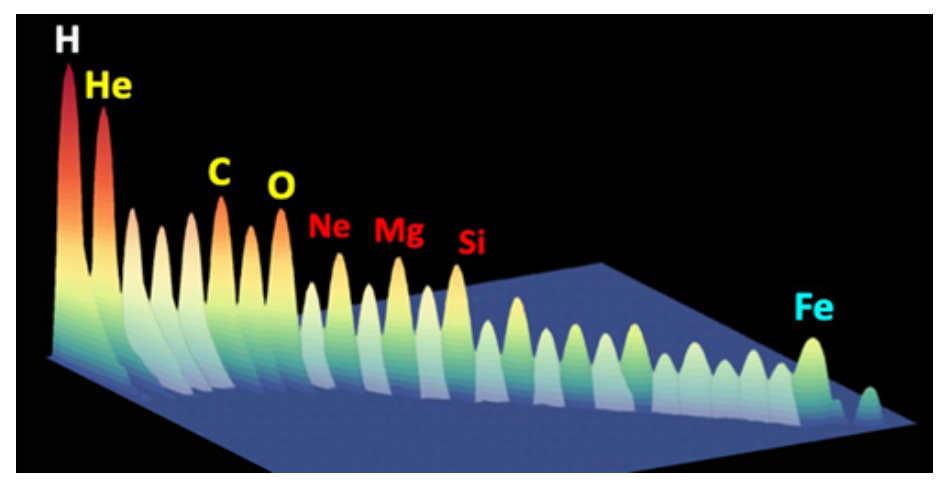

Credit: AMS Collaboration for lighter elements, such as helium, carbon, and oxygen.

With the measurement of iron (atomic number 26), the next most abundant cosmic particle after silicon, the team has reached an atomic-number frontier that won't be crossed for years to come, says Samuel Ting, a physicist at the Massachusetts Institute of Technology and lead scientist of the AMS Collaboration. The obtained spectra offer yet another puzzle because they resemble those of light species more than those of heavier ones. Ting says that big leaps in cosmic-ray modeling are now needed to make sense of the whole body of high-precision, element-specific spectra collected by the AMS.

Matteo Rini is the Editor of Physics.

\section{REFERENCES}

1. M. Aguilar et al., "Properties of iron primary cosmic rays: Results from the Alpha Magnetic Spectrometer," Phys. Rev. Lett. 126, 041104 (2021). 